\title{
Fractional nonlocal impulsive quasilinear multi-delay integro-differential systems
}

Amar Debbouche

Correspondence:

amar_debbouche@yahoo.fr Department of Mathematics,

Faculty of Science, Guelma University Guelma, Algeria

\section{SpringerOpen ${ }^{\circ}$}

\begin{abstract}
In this article, sufficient conditions for the existence result of quasilinear multi-delay integro-differential equations of fractional orders with nonlocal impulsive conditions in Banach spaces have been presented using fractional calculus, resolvent operators, and Banach fixed point theorem. As an application that illustrates the abstract results, a nonlocal impulsive quasilinear multi-delay integro-partial differential system of fractional order is given.
\end{abstract}

AMS Subject Classifications. 34K05, 34G20, 26A33, 35A05.

Keywords: Fractional integrodifferential systems, resolvent operators, nonlocal and impulsive conditions, fixed point theorem

\section{Introduction}

Many fractional models can be represented by the following system

$$
\begin{aligned}
& \frac{\mathrm{d}^{\alpha} u(t)}{\mathrm{d} t^{\alpha}}+A(t, u(t)) u(t)=f(t, u(t), u(\beta(t)))+\int_{0}^{t} g(t, s, u(s), u(\gamma(s))) \mathrm{d} s, \\
& u(0)+h(u)=u_{0}, \\
& \Delta u\left(t_{i}\right)=I_{i}\left(u\left(t_{i}\right)\right),
\end{aligned}
$$

in a Banach space $X$, where $0<\alpha \leq 1, t \in[0, a], u_{0} \in X, i=1,2, \ldots, m$ and $0<t_{1}<t_{2}<$ $\cdots<t_{m}<a$. We assume that $-A(t,$.$) is a closed linear operator defined on a dense domain$ $D(A)$ in $X$ into $X$ such that $D(A)$ is independent of $t$. It is assumed also that $-A(t,$.$) gen-$ erates an evolution operator in the Banach space $X$. The functions $f: J X^{r+1} \rightarrow X, g: \Lambda$ $\times X^{k+1} \rightarrow X, h: P C(J, X) \rightarrow X, u(\beta)=\left(u\left(\beta_{1}\right), \ldots, u\left(\beta_{r}\right)\right), u(\gamma)=\left(u\left(\gamma_{1}\right), \ldots, u\left(\gamma_{k}\right)\right)$, and $\beta_{p}, \gamma_{q}$ $: J \rightarrow J$ are given, where $p=1,2, \ldots, r$ and $q=1,2, \ldots, \mathrm{k}$. Here $J=[0, a]$ and $\Lambda=\{(t, s)$. $0 \leq s \leq t \leq a\}$. Let $P C(J, X)$ consist of functions $u$ from $J$ into $X$, such that $u(t)$ is continuous at $t \neq t_{i}$ and left continuous at $t=t_{i}$ and the right limit $u\left(t_{i}^{+}\right)$exists for $i=1$, $2, \ldots, m$. Clearly $P C(J, X)$ is a Banach space with the norm $\|u\|_{P C}=\sup _{t \in J}\|u(t)\|$, and let $\Delta u\left(t_{i}\right)=u\left(t_{i}^{+}\right)-u\left(t_{i}^{-}\right)$constitutes an impulsive condition. Fractional differential equations have proved to be valuable tools in the modelling of many phenomena in various fields of science and engineering. Indeed, we can find numerous applications in viscoelasticity, electrochemistry, control, porous media, electromagnetic, etc. (see [1-5]). They involve a wide area of applications by bringing into a broader paradigm concepts 
of physics and mathematics [6-8]. There has been a significant development in fractional differential and partial differential equations in recent years, see Kilbas et al. $[9,10]$, also in fractional nonlinear systems with delay and fractional variational principles with delay, see Baleanu et al. [11,12].

The existence results to evolution equations with nonlocal conditions in Banach space was studied first by Byszewski [13,14], subsequently, many authors were pointed in the same field, see reference therein. Deng [15] indicated that, using the nonlocal condition $u(0)+h(u)=u_{0}$ to describe for instance, the diffusion phenomenon of a small amount of gas in a transparent tube can give better result than using the usual local Cauchy problem $u(0)=u_{0}$. Let us observe also that since Deng's papers, the function $h$ is considered

$$
h(u)=\sum_{k=1}^{p} c_{k} u\left(t_{k}\right)
$$

where $c_{k}, k=1,2, \ldots, p$ are given constants and $0 \leq t_{1}<\ldots<t_{p} \leq a$. However, among the previous research on nonlocal cauchy problems, few are concerned with mild solutions of fractional semilinear differential equations, see Mophou and N'Guérékata [16], and others with fractional nonlocal boundary value problems, for instance, Ahmad et al. $[17,18]$.

The theory of impulsive differential equations has been emerging as an important area of investigation in recent years, because all the structures of its emergence have deep physical background and realistic mathematical model. The theory of impulsive differential equations appears as a natural description of several real processes subject to certain perturbations whose duration is negligible in comparison with the duration of the process. It has seen considerable development in the last decade, see the monographs of Bainov and Simeonov [19], Lakshmikantham et al. [20], and Samoilenko and Perestyuk [21] where numerous properties of their solutions are studied, and detailed bibliographies are given.

Recently, the existence of solutions of fractional abstract differential equations with nonlocal initial condition was investigated by N'Guérékata [22] and Li [23]. Much attention has been paid to existence results for the impulsive differential and integrodifferential equations of fractional order in abstract spaces, see Benchohra et al. [2,24]. Several authors have studied the existence of solutions of abstract quasilinear evolution equations in Banach space [25-27].

Regarding this article, it generalizes previous results concerned the existence of solutions to nonlocal and impulsive integrodifferential equations of quasilinear type with delays of arbitrary orders. Section "Preliminaries" is devoted to a review of some essential results. In next section, we state and prove our main results, the last section deals to giving an example to illustrate the abstract results.

\section{Preliminaries}

Let $X$ and $Y$ be two Banach spaces such that $Y$ is densely and continuously embedded in $X$. For any Banach space $Z$, the norm of $Z$ is denoted by $\|\cdot\|_{Z}$. The space of all bounded linear operators from $X$ to $Y$ is denoted by $B(X, Y)$ and $B(X, X)$ is written as $B(X)$. We recall some definitions in fractional calculus from Gelfand-Shilov [28] and Podlubny [29], then some known facts of the theory of semigroups from Pazy [30]. 
Definition 2.1 The fractional integral of order with the lower limit zero for a function $f \in C([0, \infty))$ is defined as

$$
I^{\alpha} f(t)=\frac{1}{\Gamma(\alpha)} \int_{0}^{t} \frac{f(s)}{(t-s)^{1-\alpha}} \mathrm{d} s, \quad t>0,0<\alpha<1,
$$

provided the right side is pointwise defined on $[0, \infty)$, where $\Gamma$ is the gamma function. Riemann-Liouville derivative of order $\alpha$ with the lower limit zero for a function $f$ $\in C([0, \infty))$ can be written as

$$
{ }^{L} D^{\alpha} f(t)=\frac{1}{\Gamma(1-\alpha)} \frac{\mathrm{d}}{\mathrm{d} t} \int_{0}^{t} \frac{f(s)}{(t-s)^{\alpha}} \mathrm{d} s, \quad t>0,0<\alpha<1 .
$$

The Caputo derivative of order for a function $f \in C([0, \infty))$ can be written as

$$
{ }^{C} D^{\alpha} f(t)={ }^{L} D^{\alpha}(f(t)-f(0)), \quad t>0,0<\alpha<1 .
$$

\section{Remark 2.1}

(1) If $f \in C^{1}([0, \infty))$, then

$$
{ }^{C} D^{\alpha} f(t)=\frac{1}{\Gamma(1-\alpha)} \int_{0}^{t} \frac{f^{\prime}(s)}{(t-s)^{\alpha}} \mathrm{d} s=I^{1-\alpha} f^{\prime}(t), \quad t>0,0<\alpha<1 .
$$

(2) The Caputo derivative of a constant is equal to zero.

(3) If $f$ is an abstract function with values in $X$, then integrals which appear in Definition 2.1 are taken in Bochner's sense.

Definition 2.2 A two parameter family of bounded linear operators $U(t, s), 0 \leq s \leq t$ $\leq a$, on $X$ is called an evolution system if the following two conditions are satisfied

(i) $U(t, t)=I, U(t, r) U(r, s)=U(t, s)$ for $0 \leq s \leq r \leq t \leq a$,

(ii) $(t, s) \rightarrow U(t, s)$ is strongly continuous for $0 \leq s \leq t \leq a$.

More detail about evolution system and quasilinear equation of evolution can be found in [30, Chap. 5 and Sect. 6.4, respectively].

Let $E$ be the Banach space formed from $D(A)$ with the graph norm. Since - $A(t)$ is a closed operator, it follows that $-A(t)$ is in the set of bounded operators from $E$ to $X$.

Definition 2.3 [31-33] A resolvent operators for problem (1.1)-(1.3) is a bounded operators valued function $R_{u}(t, s) \in B(X), 0 \leq s \leq t \leq a$, the space of bounded linear operators on $X$, having the following properties:

(i) $R_{u}(t, s)$ is strongly continuous in $s$ and $t, R_{u}(s, s)=I, 0 \leq s \leq a,\left\|R_{u}(t, s)\right\| \leq M e^{N}$

${ }^{(t, s)}$ for some constants $M$ and $N$.

(ii) $R_{u}(t, s) E \subset E, R_{u}(t, s)$ is strongly continuous in $s$ and $t$ on $E$.

(iii) For $x \in X, R_{u}(t, s) x$ is continuously differentiable in $s \in[0, a]$ and

$$
\frac{\partial R_{u}}{\partial s}(t, s) x=R_{u}(t, s) A(s, u(s)) x .
$$

(iv) For $x \in X$ and $s \in[0, a], R_{u}(t, s) x$ is continuously differentiable in $t \in[s, a]$ and

$$
\frac{\partial R_{u}}{\partial t}(t, s) x=-A(t, u(t)) R_{u}(t, s) x,
$$


with $\frac{\partial R_{u}}{\partial s}(t, s) x$ and $\frac{\partial R_{u}}{\partial t}(t, s) x$ are strongly continuous on $0 \leq s \leq t \leq a$. Here $R_{u}(t, s)$ can be extracted from the evolution operator of the generator - $A(t, u)$. The resolvent operator is similar to the evolution operator for nonautonomous differential equations in a Banach space. Let $\Omega$ be a subset of $X$.

Definition 2.4 (Compare [31] with [7,22,34]) By a mild solution of (1.1)-(1.3) we mean a function $u \in P C(J: X)$ with values in $\Omega$ satisfying the integral equation

$$
\begin{aligned}
u(t)=R_{u}( & (, 0) u_{0}-R_{u}(t, 0) h(u) \\
& +\frac{1}{\Gamma(\alpha)} \int_{0}^{t}(t-s)^{\alpha-1} R_{u}(t, s)\left[f(s, u(s), u(\beta(s)))+\int_{0}^{s} g(s, \eta, u(\eta), u(\gamma(\eta))) \mathrm{d} \eta\right] \mathrm{d} s \\
& +\sum_{0<t_{i}<t} R_{u}\left(t, t_{i}\right) I_{i}\left(u\left(t_{i}\right)\right), t \in J
\end{aligned}
$$

for all $\mathrm{u}_{0} \in X$.

Definition 2.5 (Compare [35,36] with [2]) By a classical solution of (1.1)-(1.3) on J, we mean a function $u$ with values in $X$ such that:

(1) $u$ is continuous function on $J \backslash\left\{t_{1}, t_{2}, \ldots, t_{m}\right\}$ and $u(t) \in D(A)$,

(2) $\frac{\mathrm{d}^{\alpha} u}{\mathrm{~d} t^{\alpha}}$ exists and continuous on $J_{0}, 0<\alpha<1$,

(3) $u$ satisfies (1.1) on $J_{0}$, the nonlocal condition (1.2) and the impulsive condition

(1.3), where $J_{0}=(0, a] \backslash\left\{t_{1}, t_{2}, \ldots, t_{m}\right\}$. We assume the following conditions

$\left(\mathrm{H}_{1}\right) h: P C(J: \Omega) \rightarrow Y$ is Lipschitz continuous in $X$ and bounded in $Y$, i.e., there exist constants $k_{1}>0$ and $k_{2}>0$ such that

$$
\begin{gathered}
\|h(u)\|_{Y} \leq k_{1}, \\
\|h(u)-h(v)\|_{Y} \leq k_{2} \max _{t \in J}\|u-v\|_{P C}, \quad u, v \in P C(J: X) .
\end{gathered}
$$

For the conditions $\left(\mathrm{H}_{2}\right)$ and $\left(\mathrm{H}_{3}\right)$ let $\mathrm{Z}$ be taken as both $\times$ and $\mathrm{Y}$.

$\left(\mathrm{H}_{2}\right) g: \Lambda \times Z^{k+1} \rightarrow Z$ is continuous and there exist constants $k_{3}>0$ and $k_{4}>0$ such that

$$
\begin{gathered}
\int_{0}^{t}\left\|g\left(t, s, u_{1}, \ldots, u_{k+1}\right)-g\left(t, s, v_{1}, \ldots, v_{k+1}\right)\right\|_{Z} \mathrm{~d} s \leq k_{3} \sum_{q=1}^{k+1}\left\|u_{q}-v_{q}\right\|_{Z}, \quad u_{q}, v_{q} \in X, \quad q=1, \ldots, k+1, \\
k_{4}=\max \left\{\int_{0}^{t}\|g(t, s, 0, \ldots, 0)\|_{Z} \mathrm{~d} s:(t, s) \in \Lambda\right\} .
\end{gathered}
$$

$\left(\mathrm{H}_{3}\right) f: J \times \mathrm{Z}^{r+1} \rightarrow Z$ is continuous and there exist constants $k_{5}>0$ and $k_{6}>0$ such that

$$
\begin{gathered}
\left\|f\left(t, u_{1}, \ldots, u_{r+1}\right)-f\left(t, v_{1}, \ldots, v_{r+1}\right)\right\| z \leq k_{5} \sum_{p=1}^{r+1}\left\|u_{p}-v_{p}\right\|_{Z}, \quad u_{p}, v_{p} \in X, \quad p=1, \ldots, r+1, \\
k_{6}=\max _{t \in J}\|f(t, 0, \ldots, 0)\|_{z} .
\end{gathered}
$$

$\left(\mathrm{H}_{4}\right) \beta_{p}, \gamma_{q}: J \rightarrow J$ are bijective absolutely continuous and there exist constants $c_{p}>0$ and $b_{q}>0$ such that $\beta_{p}^{\prime}(t) \geq c_{p}$ and $\gamma_{q}^{\prime}(t) \geq b_{q}$, respectively, for $t \in J, p=1, \ldots, r$ and $q=$ $1, \ldots, k$.

$\left(\mathrm{H}_{5}\right) I_{i}: X \rightarrow X$ are continuous and there exist constants $l_{i}>0, i=1,2, \ldots, m$ such that

$$
\left\|I_{i}(u)-I_{i}(v)\right\| \leq l_{i}\|u-v\|, \quad u, v \in X .
$$


Let us take $M_{0}=\max \left\|R_{u}(t, s)\right\|_{B(Z)}, 0 \leq s \leq t \leq a, u \in \Omega$.

$\left(\mathrm{H}_{6}\right)$ There exist positive constants $\delta_{1}, \delta_{2}, \delta_{3} \in(0, \delta / 3]$ and $\lambda_{1}, \lambda_{2}, \lambda_{3} \in\left[0, \frac{1}{3}\right)$ such that

$$
\delta_{1}=M_{0}\left\|u_{0}\right\|_{Y}+M_{0} k_{1}, \quad \delta_{2}=M_{0} \theta, \quad \delta_{3}=M_{0} \xi,
$$

and

$$
\begin{aligned}
& \lambda_{1}=K a\left\|u_{0}\right\|_{Y}+k_{1} K a+M_{0} k_{2}, \\
& \lambda_{2}=K a \theta+M_{0} \sigma\left[k_{5}\left(1+1 / c_{1}+\cdots+1 / c_{r}\right)+k_{3}\left(1+1 / b_{1}+\cdots+1 / b_{k}\right)\right], \\
& \lambda_{3}=K a \xi+M_{0} \sum_{i=1}^{m} l_{i,}
\end{aligned}
$$

where $\rho=\sigma\left[k_{5}\left(1 / c_{1}+\cdots+1 / c_{r}\right)+k_{3}\left(1 / b_{1}+\cdots+1 / b_{k}\right)\right], \theta=\sigma \delta\left(k_{3}+k_{5}\right)+\rho \delta+\sigma\left(k_{4}+\right.$ $\left.k_{6}\right), \sigma=\frac{a^{\alpha}}{\Gamma(1+\alpha)}$ and $\xi=\sum_{i=1}^{m}\left(l_{i} \delta+\left\|I_{i}(0)\right\|\right)$.

\section{Main results}

Lemma 3.1 Let $R_{u}(t, s)$ the resolvent operators for the fractional problem (1.1)-(1.3). There exists a constant $K>0$ such that

$$
\left\|R_{u}(t, s) \omega-R_{v}(t, s) \omega\right\| \leq K\|\omega\|_{Y} \int_{s}^{t}\|u(\tau)-v(\tau)\| \mathrm{d} \tau,
$$

for every $u, v \in P C(J: X)$ with values in $\Omega$ and every $\omega \in Y$, see [30, lemma 4.4, p. 202].

Let $S_{\delta}=\left\{u: u \in P C(J: X), u(0)+h(u)=u_{0}, \Delta u\left(t_{\mathrm{i}}\right)=I_{i}\left(u\left(t_{i}\right)\right),\|u\| \leq \delta\right\}$, for $t \in J, \delta>$ $0, u_{0} \in X$ and $i=1, \ldots, m$.

Lemma 3.2

$$
\|\varphi(t)\|_{Y} \leq \theta,
$$

where

$$
\varphi(t)=\frac{1}{\Gamma(\alpha)} \int_{0}^{t}(t-s)^{\alpha-1}\left[f(s, u(s), u(\beta(s)))+\int_{0}^{s} g(s, \tau, u(\tau), u(\gamma(\tau))) \mathrm{d} \tau\right] \mathrm{d} s .
$$

Proof We have

$$
\begin{aligned}
& \|\varphi(t)\|_{Y} \\
& \leq \frac{1}{\Gamma(\alpha)} \int_{0}^{t}(t-s)^{\alpha-1}\left[\| f\left(s, u(s), u\left(\beta_{1}(s)\right), \ldots, u\left(\beta_{r}(s)\right)-f(s, 0, \ldots, 0)\|+\| f(s, 0, \ldots, 0) \|\right.\right. \\
& \quad+\int_{0}^{s} \| g\left(s, \tau, u(\tau), u\left(\gamma_{1}(\tau)\right), \ldots, u\left(\gamma_{k}(\tau)\right)-g(s, \tau, 0, \ldots, 0)\left\|\mathrm{d} \tau+\int_{0}^{s}\right\| g(s, \tau, 0, \ldots, 0) \| \mathrm{d} \tau\right] \mathrm{d} s .
\end{aligned}
$$


Using $\mathrm{H}_{2}, \mathrm{H}_{3}$, and $\mathrm{H}_{4}$, we get

$$
\begin{aligned}
\|\varphi(t)\|_{Y} & \leq \frac{1}{\Gamma(\alpha)} \int_{0}^{t}(t-s)^{\alpha-1}\left[k_{5}\left(\|u(s)\|+\left\|u\left(\beta_{1}(s)\right)\right\|+\cdots+\left\|u\left(\beta_{r}(s)\right)\right\|\right)+k_{6}\right. \\
& \left.+k_{3}\left(\|u(s)\|+\left\|u\left(\gamma_{1}(s)\right)\right\|+\cdots+\left\|u\left(\gamma_{k}(s)\right)\right\|\right)+k_{4}\right] \mathrm{d} s \\
\leq & \frac{1}{\Gamma(\alpha)} \int_{0}^{t}(t-s)^{\alpha-1}\left[k_{5}\left\{\delta+\left\|u\left(\beta_{1}(s)\right)\right\|\left(\beta_{1}^{\prime}(s) / c_{1}\right)+\cdots+\left\|u\left(\beta_{r}(s)\right)\right\|\left(\beta_{r}^{\prime}(s) / c_{r}\right)\right\}+k_{6}\right. \\
& \left.+k_{3}\left\{\delta+\left\|u\left(\gamma_{1}(s)\right)\right\|\left(\gamma_{1}^{\prime}(s) / b_{1}\right)+\cdots+\left\|u\left(\gamma_{k}(s)\right)\right\|\left(\gamma_{k}^{\prime}(s) / b_{k}\right)\right\}+k_{4}\right] \mathrm{d} s \\
\leq & \sigma \delta\left(k_{3}+k_{5}\right)+\sigma\left(k_{4}+k_{6}\right) \\
& +\frac{k_{5}}{c_{1} \Gamma(\alpha)} \int_{\beta_{1}(0)}^{\beta_{1}(t)}\left(t-\beta_{1}^{-1}(\tau)\right)^{\alpha-1}\|u(\tau)\| d \tau+\cdots+\frac{k_{5}}{c_{r} \Gamma(\alpha)} \int_{\beta_{r}(0)}^{\beta_{r}(t)}\left(t-\beta_{r}^{-1}(\tau)\right)^{\alpha-1}\|u(\tau)\| \mathrm{d} \tau \\
& +\frac{k_{3}}{b_{1} \Gamma(\alpha)} \int_{\gamma_{1}(0)}^{\gamma_{1}(t)}\left(t-\gamma_{1}^{-1}(\eta)\right)^{\alpha-1}\|u(\eta)\| \mathrm{d} \eta+\cdots+\frac{k_{3}}{b_{k} \Gamma(\alpha)} \int_{\gamma_{k}(0)}^{\gamma_{k}}\left(t-\gamma_{k}^{-1}(\eta)\right)^{\alpha-1}\|u(\eta)\| \mathrm{d} \eta .
\end{aligned}
$$

Hence the required result.

Theorem 3.3 Suppose that the operator $-A(t, u)$ generates the resolvent operator $R_{u}$ $(t, s)$ with $\left\|R_{u}(t, s)\right\| \leq M e^{N(t-s)}$. If the hypotheses $\left(\mathrm{H}_{1}\right)-\left(\mathrm{H}_{6}\right)$ are satisfied, then the fractional integro-differential equation (1.1) with nonlocal condition (1.2) and impulsive condition (1.3) has a unique mild solution on $J$ for all $u_{0} \in X$.

Proof Consider a mapping $P$ on $S_{\delta}$ defined by

$(P u)(t)=R_{u}(t, 0) u_{0}-R_{u}(t, 0) h(u)$

$$
\begin{aligned}
& +\frac{1}{\Gamma(\alpha)} \int_{0}^{t}(t-s)^{\alpha-1} R_{u}(t, s)\left[f(s, u(s), u(\beta(s)))+\int_{0}^{s} g(s, \eta, u(\eta), u(\gamma(\eta))) \mathrm{d} \eta\right] \mathrm{d} s \\
& +\sum_{0<t_{i}<t} R_{u}\left(t, t_{i}\right) I_{i}\left(u\left(t_{i}\right)\right) .
\end{aligned}
$$

We shall show that $P: S_{\delta} \rightarrow S_{\delta}$. For $u \in S_{\delta}$, we have

$$
\begin{aligned}
\|P u(t)\| y \leq & \left\|R_{u}(t, 0) u_{0}\right\|+\left\|R_{u}(t, 0) h(u)\right\| \\
& +\left\|\frac{1}{\Gamma(\alpha)} \int_{0}^{t}(t-s)^{\alpha-1} R_{u}(t, s)\left[f(s, u(s), u(\beta(s)))+\int_{0}^{s} g(s, \eta, u(\eta), u(\gamma(\eta))) \mathrm{d} \eta\right] \mathrm{d} s\right\| \\
& +\sum_{0<t_{i}<t}\left\|R_{u}\left(t, t_{i}\right)\right\|\left(\left\|I_{i}\left(u\left(t_{i}\right)\right)-I_{i}(0)\right\|+\left\|I_{i}(0)\right\|\right) .
\end{aligned}
$$

Using $\mathrm{H}_{1}$, Lemma 3.2 and $\mathrm{H}_{5}$, we get

$$
\left\|P u(t)_{Y}\right\| \leq M_{0}\left\{\left\|u_{0}\right\|+k_{1}+\theta+\sum_{i=1}^{m}\left(l_{i} \delta+\left\|I_{i}(0)\right\|\right)\right\} .
$$

From assumption $\mathrm{H}_{6}$, one gets $\left\|\left(P u_{\mu}\right)(t)\right\|_{Y} \leq \delta$. Thus, $P$ maps $S_{\delta}$ into itself. Now for $u, v \in S_{\delta}$, we have

$$
\|P u(t)-P v(t)\| \leq I_{1}+I_{2}+I_{3},
$$

where

$$
\begin{aligned}
I_{1}= & \left\|R_{u}(t, 0) u_{0}-R_{v}(t, 0) u_{0}\right\|+\left\|R_{u}(t, 0) h(u)-R_{v}(t, 0) h(v)\right\|, \\
I_{2}= & \frac{1}{\Gamma(\alpha)} \int_{0}^{t}(t-s)^{\alpha-1} \| R_{u}(t, s)\left[f(s, u(s), u(\beta(s)))+\int_{0}^{s} g(s, \eta, u(\eta), u(\gamma(\eta))) \mathrm{d} \eta\right] \\
& \quad-R_{\nu}(t, s)\left[f(s, v(s), v(\beta(s)))+\int_{0}^{s} g(s, \eta, v(\eta), v(\gamma(\eta))) \mathrm{d} \eta\right] \| \mathrm{d} s
\end{aligned}
$$


and

$$
I_{3}=\sum_{i=1}^{m}\left\|R_{u}\left(t, t_{i}\right) I_{i}\left(u\left(t_{i}\right)\right)-R_{v}\left(t, t_{i}\right) I_{i}\left(v\left(t_{i}\right)\right)\right\| .
$$

Applying Lemma 3.1 and $\mathrm{H}_{1}$, we get

$$
\begin{aligned}
I_{1} \leq & \left\|R_{u}(t, 0) u_{0}-R_{v}(t, 0) u_{0}\right\|+\left\|R_{u}(t, 0) h(u)-R_{v}(t, 0) h(u)\right\| \\
& +\left\|R_{v}(t, 0) h(u)-R_{v}(t, 0) h(v)\right\| \\
\leq & \left\{K a\left\|u_{0}\right\|_{Y}+k_{1} K a+M_{0} k_{2}\right\} \max _{\tau \in J}\|u(\tau)-v(\tau)\| .
\end{aligned}
$$

Also, we apply Lemmas $3.1,3.2, \mathrm{H}_{2}, \mathrm{H}_{3}, \mathrm{H}_{4}$, and $\mathrm{H}_{6}$, we obtain

$$
\begin{aligned}
I_{2} \leq & \frac{1}{\Gamma(\alpha)} \int_{0}^{t}(t-s)^{\alpha-1}\left\{\| R_{u}(t, s)\left[f(s, u(s), u(\beta(s)))+\int_{0}^{s} g(s, \eta, u(\eta), u(\gamma(\eta))) \mathrm{d} \eta\right]\right. \\
- & R_{v}(t, s)\left[f(s, u(s), u(\beta(s)))+\int_{0}^{s} g(s, \eta, u(\eta), u(\gamma(\eta))) \mathrm{d} \eta\right] \| \\
+ & R_{v}(t, s)\left[f(s, u(s), u(\beta(s)))+\int_{0}^{s} g(s, \eta, u(\eta), u(\gamma(\eta))) \mathrm{d} \eta\right] \\
& -R_{v}(t, s)\left[f(s, v(s), v(\beta(s)))+\int_{0}^{s} g(s, \eta, v(\eta), v(\gamma(\eta))) \mathrm{d} \eta\right] \| \mathrm{d} s \\
\leq & K a \theta \max _{\tau \in J}\|u(\tau)-v(\tau)\| \\
+ & M_{0} \frac{1}{\Gamma(\alpha)} \int_{0}^{t}(t-s)^{\alpha-1}\left\{k_{5}\left[\|u(s)-v(s)\|+\sum_{p=1}^{r}\left\|u\left(\beta_{p}(s)\right)-v\left(\beta_{p}(s)\right)\right\|\left(\beta_{p}^{\prime}(s) / c_{p}\right)\right]\right. \\
& \left.+k_{3}\left[\|u(s)-v(s)\|+\sum_{q=1}^{k}\left\|u\left(\gamma_{q}(s)\right)-v\left(\gamma_{q}(s)\right)\right\|\left(\gamma_{q}^{\prime}(s) / b_{q}\right)\right]\right\} \mathrm{d} s \\
\leq & K a \theta \max _{\tau \in J}\|u(\tau)-v(\tau)\| \\
+ & M_{0} \sigma\left[k_{5}\left(1+1 / c_{1}+\cdots+1 / c_{r}\right)+k_{3}\left(1+1 / b_{1}+\cdots+1 / b_{k}\right)\right] \max _{\tau \in J}\|u(\tau)-v(\tau)\| .
\end{aligned}
$$

Again, Lemma 3.1, $\mathrm{H}_{5}$ and $\mathrm{H}_{6}$, we have

$$
\begin{aligned}
I_{3} & \leq \sum_{i=1}^{m}\left\{\left\|R_{u}\left(t, t_{i}\right) I_{i}\left(u\left(t_{i}\right)\right)-R_{v}\left(t, t_{i}\right) I_{i}\left(u\left(t_{i}\right)\right)\right\|+\left\|R_{v}\left(t, t_{i}\right) I_{i}\left(u\left(t_{i}\right)\right)-R_{v}\left(t, t_{i}\right) I_{i}\left(v\left(t_{i}\right)\right)\right\|\right\} \\
& \leq\left\{K \sum_{i=1}^{m}\left(l_{i} \delta+\left\|I_{i}(0)\right\|\right) a+M_{0} \sum_{i=1}^{m} l_{i}\right\} \max _{\tau \in J}\|u(\tau)-v(\tau)\| .
\end{aligned}
$$

It follows from these estimations that

$$
\|P u(t)-P v(t)\| \leq \lambda \max _{\tau \in J}\|u(\tau)-v(\tau)\|,
$$

where $0 \leq \lambda<1$. Thus $P$ is a contraction on $S_{\delta}$. From the contraction mapping theorem, $P$ has a unique fixed point $u \in S_{\delta}$ which is the mild solution of (1.1)-(1.3) on $J$.

Theorem 3.4 Assume that

(i) Conditions $\left(\mathrm{H}_{1}\right)-\left(\mathrm{H}_{6}\right)$ hold,

(ii) $\mathrm{Y}$ is a reflexive Banach space with norm $\|\cdot\|$,

(iii) The functions $f$ and $g$ are uniformly Hölder continuous in $t \in J$. 
Then the problem (1.1)-(1.3) has a unique classical solution on $J$.

Proof From (i), applying Theorem 3.3, the problem (1.1)-(1.3) has a unique mild solution $u \in S_{\delta}$. Set

$$
\omega(t)=f(t, u(t), u(\beta(t)))+\int_{0}^{t} g(t, s, u(s), u(\gamma(s))) \mathrm{d} s .
$$

In order to prove the regularity of the mild solution, we use the further assumptions, it is easy to conclude that the function $\omega(t)$ is also uniformly Hölder continuous in $t \in$ $J$. Consider the following fractional differential equation

$$
\frac{\mathrm{d}^{\alpha} v(t)}{\mathrm{d} t^{\alpha}}+A(t, u) u(t)=\omega(t)
$$

with the nonlocal condition (1.2) and impulsive condition (1.3).

According to Pazy [30], the late problem has a unique solution $v$ on $J$ into $X$ given by

$$
\begin{aligned}
v(t)=R_{u}(t, 0) u_{0}-R_{u}(t, 0) h(u)+\frac{1}{\Gamma(\alpha)} \int_{0}^{t}(t-s)^{\alpha-1} R_{u}(t, s) \omega(s) \mathrm{d} s \\
+\sum_{0<t_{i}<t} R_{u}\left(t, t_{i}\right) I_{i}\left(u\left(t_{i}\right)\right) .
\end{aligned}
$$

Noting that, each term on the right-hand side belongs to $D(A)$, using the uniqueness of $\mathrm{v}(\mathrm{t})$, we have that $u(t) \in D(A)$. It follows that $u$ is a unique classical solution of (1.1)-(1.3) on $J$.

\section{Application}

Consider the nonlinear integro-partial differential equation of fractional order

$$
\begin{aligned}
& \frac{\partial^{\alpha} u(x, t)}{\partial t^{\alpha}}+\sum_{|q| \leq 2 m} a_{q}(x, t) u(x, t) D_{x}^{q} u(x, t)=F\left(x, t, u, w_{1}\right)+\int_{0}^{t} G\left(x, t, s, u(x, s), w_{2}(s)\right) \mathrm{d} s, \\
& u(x, 0)+\sum_{k=1}^{p} c_{k} u\left(x, t_{k}\right)=g(x) \\
& \Delta u\left(x, t_{k}\right)=\int_{R^{n}} \rho k(y, x) u\left(y, t_{k}\right) \mathrm{d} y
\end{aligned}
$$

where $0<\alpha \leq 1,0 \leq t_{1}<\cdots<t_{p} \leq a, x \in R^{n}, D_{x}^{q}=D_{x_{1}}^{q_{1}} \ldots D_{x_{n}}^{q_{n}}, D_{x_{i}}=\frac{\partial}{\partial x_{i}}, q=\left(q_{1}, \ldots, q_{n}\right)$ is an $n$-dimensional multi-index, $|q|=q_{1}+\cdots+q_{n}$, and $w_{i}, i=1$, 2 , is given by

$$
w_{i}(x, t)=\sum_{|q| \leq 2 m-1} b_{q i}(x, t) D_{x}^{q} u(x, \sin t)+\int_{\Omega} \sum_{|q| \leq 2 m-1} c_{q_{i}}(x, t) D_{y}^{q} u(y, \sin t) \mathrm{d} y .
$$

Let $\mathrm{L}_{2}\left(R^{n}\right)$ be the set of all square integrable functions on $R^{n}$. We denote by $C^{m}\left(R^{n}\right)$ the set of all continuous real-valued functions defined on $R^{n}$ which have continuous partial derivatives of order less than or equal to $m$. By $C_{0}^{m}\left(R^{n}\right)$ we denote the set of all 
functions $f \in C^{m}\left(R^{n}\right)$ with compact supports. Let $H^{m}\left(R^{n}\right)$ be the completion of $C_{0}^{m}\left(R^{n}\right)$ with respect to the norm

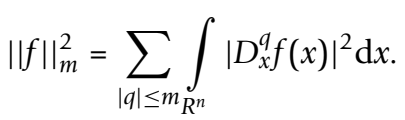

It is supposed that

(i) The operator $A(t, u)=-\sum_{|q| \leq 2 m} a_{q}(x, t) u(x, t) D_{x}^{q}$ is uniformly elliptic on $R^{n}$. In other words, all the coefficients $a_{q},|q|=2 m$, are continuous and bounded on $R^{n}$ and there is a positive number $c$ such that

$$
(-1)^{m+1} \sum_{|q|=2 m} a_{q}(x, t) u(x, t) \xi^{q} \geq c|\xi|^{2 m},
$$

for all $x L R^{n}$ and all $\xi \neq 0, \xi \in R^{n}, \xi^{q}=\xi_{1}^{q 1} \ldots \xi_{n^{n}}^{q}$ and $|\xi|^{2}=\xi_{1}^{2}+\ldots+\xi_{n}^{2}$.

(ii) All the coefficients $a_{q},|q|=2 m$, satisfy a uniform Hölder condition on $R^{n}$. Under these conditions the operator $A$ with domain of definition $D(A)=H^{2 m}\left(R^{n}\right)$ generates an evolution operator defined on $L_{2}\left(R^{n}\right)$, and it is well known that $H^{2 m}\left(R^{n}\right)$ is dense in $X=L_{2}\left(R^{n}\right)$ and the initial function $g(x)$ is an element in Hilbert space $H^{2 m}\left(R^{n}\right)$, see $[14,15,35]$. Applying Theorem 3.3, this achieves the proof of the existence of mild solutions of the system (4.1)-(4.3). In addition,

(iii) If the coefficients $b_{q}, c_{q},|q| \leq 2 m$ - 1 satisfy a uniform Hölder condition on $R^{n}$ and the operators $F$ and $G$ satisfy

There are numbers $L_{1}, L_{2} \geq 0$ and $\lambda_{1}, \lambda_{2} L(0,1)$ such that

$$
\sum_{|q| \leq 2 m-1} \int_{R^{n}}\left|F\left(x, t, u, D_{x}^{q} w_{1}\right)-F\left(x, s, u, D_{x}^{q} w_{1}^{*}\right)\right|^{2} \mathrm{~d} x \leq L_{1}\left(|t-s|^{\lambda_{1}}+\left|w_{1}-w_{1}^{*}\right|^{2} \mathrm{~d} x\right) .
$$

and

$$
\sum_{|q| \leq 2 m-1} \int_{R^{n}}\left|G\left(x, t, \eta, u, D_{x}^{q} w_{2}\right)-G\left(x, s, \eta, u, D_{x}^{q} w_{2}\right)\right|^{2} \mathrm{~d} x \leq L_{2}|t-s|^{\lambda_{2}} .
$$

for all $t, s \in I,(t, \eta),(s, \eta) L \Delta$, and all $x \in R^{n}$. Applying Theorem 3.4, we deduce that (4.1)-(4.3) has a unique strong solution.

\section{Competing interests}

The author declare that he has no competing interests.

Received: 15 December 2010 Accepted: 24 May 2011 Published: 24 May 2011

\section{References}

1. Agrawal OP, Defterli O, Baleanu D: Fractional optimal control problems with several state and control variables. J Vibr Control 2010, 16(13):1967-1976.

2. Benchohra M, Slimani BA: Existence and uniqueness of solutions to impulsive fractional differential equations. Electron J Diff Eqns 2009, 10:1-11.

3. Hilfer R: Applications of fractional calculus in physics. World Scientific, Singapore 2000.

4. Li F, N'Guerekata GM: Existence and uniqueness of mild solution for fractional integrodifferential equations. Adv Differ Equ 2010, 10, Article ID 158789.

5. Oldham KB, Spanier J: The fractional calculus. Academic Press, New York, London; 1974.

6. Agarwal RP, Lakshmikantham V, Nieto JJ: On the concept of solution for fractional differential equations with uncertainty. Nonlinear Anal 2010, 72:2859-2862.

7. Balachandran K, Trujillo JJ: The nonlocal Cauchy problem for nonlinear fractional integrodifferential equations in Banach spaces. Nonlinear Anal 2010, 72:4587-4593.

8. Baleanu D, Trujillo J: A new method of finding the fractional Euler-Lagrange and Hamilton equations within Caputo fractional derivatives. Commun Nonlinear Sci Numer Simul 2010, 15(5):1111-1115. 
9. Kilbas AA, Srivastava HM, Trujillo JJ: Theory and applications of fractional differential Equations. In North-Holland Mathematics Studies. Volume 204. Elsevier, Amsterdam; 2006.

10. Samko SG, Kilbas AA, Marichev Ol: Fractional integrals and derivatives: Theory and applications, Gordon and Breach, Yverdon 1993.

11. Baleanu D, Maaraba T, Jarad F: Fractional variational principles with delay. J Phys A 2008, 41(31), Article Number 315403.

12. Sadati SJ, Baleanu D, Ranjbar A, Ghaderi R, Abdeljawad T: Mittag-Leffler stability theorem for fractional nonlinear systems with delay. Abstr Appl Anal 2010, 7, Article ID 108651.

13. Byszewski L: Theorems about the existence and uniqueness of solutions of a semilinear evolution nonlocal Cauchy problem. J Math Anal Appl 1991, 162:494-505.

14. Byszewski L: Theorems about the existence and uniqueness of continuous solutions of nonlocal problem for nonlinear hyperbolic equation. Appl Anal 1991, 40:173-180.

15. Deng K: Exponential decay of solutions of semilinear parabolic equations with nonlocal initial conditions. J Math Annal Appl 1993, 179:630-637.

16. Mophou GM, N'Guérékata GM: A note on a semilinear fractional differential equation of neutral type with infinite delay. Adv Differ Equ 2010, 8, Article ID 674630

17. Ahmad B, Sivasundaram S: On four-point nonlocal boundary value problems of non-linear integro-differential equations of fractional order. Appl Math Comput 2010, 217:480-487.

18. Ahmad B, Nieto Jj: Existence of solutions for nonlocal boundary value problems of higher order nonlinear fractional differential equations. Abstr Appl Anal 2009, 9, Article ID 494720.

19. Bainov DD, Simeonov PS: Systems with impulse effect. Ellis Horwood Ltd., Chichister; 1989.

20. Lakshmikantham V, Bainov DD, Simeonov PS: Theory of impulsive differential equations. World Scientific, Singapore 1989.

21. Samoilenko AM, Perestyuk NA: Impulsive differential equations. World Scientific, Singapore 1995.

22. N'Guerekata GM: A Cauchy problem for some fractional abstract differential equation with non local conditions. Nonlinear Anal 2009, 70:1873-1876.

23. Li F: Mild solutions for fractional differential equations with nonlocal conditions. Adv Differ Equ 2010, 9, Article ID 287861.

24. Benchohra M, Gatsori EP, G'Orniewicz L, Ntouyas SK: Nondensely defined evolution impulsive differential equations with nonlocal conditions. Fixed Point Theory 2003, 4(2):185-204.

25. Amann H: Quasilinear evolution equations and parabolic systems. Trans Am Math Soc 1986, 29:191-227.

26. Dong Q, Li G, Zhang J: Quasilinear nonlocal integrodifferential equations in Banach spaces. Electron J Diff Equ 2008, 19:1-8.

27. Sanekata N: Abstract quasilinear equations of evolution in nonreflexive Banach spaces. Hiroshima Math J 1989, 19:109-139.

28. Gelfand IM, Shilov GE: Generalized functions. Moscow, Nauka; 19591.

29. Podlubny I: Fractional differential equations, Mathematics in Science and Engineering. Technical University of Kosice, Slovak Republic; 1999198

30. Pazy A: Semigroups of linear operators and applications to partial differential equations. Springer, Berlin; 1983.

31. Debbouche A: Fractional evolution integro-differential systems with nonlocal conditions. Adv Dyn Syst Appl 2010, 5(1):49-60

32. Sakthivel R, Choi QH, Anthoni SM: Controllability result for nonlinear evolution integrodifferential systems. App/ Math Lett 2004, 17:1015-1023.

33. Sakthivel R, Anthoni SM, Kim JH: Existence and controllability result for semilinear evolution integrodifferential systems. Math Comput Model 2005, 41:1005-1011.

34. Yan Z: Existence of solutions for nonlocal impulsive partial functional integrodifferential equations via fractional operators. Journal of Computational and Applied Math-ematics 2011, 235(8):2252-2262.

35. Debbouche A, El-Borai MM: Weak almost periodic and optimal mild solutions of fractional evolution equations. Electron J Diff EqU 2009, 46:1-8.

36. El-Borai MM, Debbouche A: On some fractional integro-differential equations with analytic semigroups. Int J C Math Sci 2009, 4(28):1361-1371.

doi:10.1186/1687-1847-2011-5

Cite this article as: Debbouche: Fractional nonlocal impulsive quasilinear multi-delay integro-differential systems. Advances in Difference Equations 2011 2011:5.

\section{Submit your manuscript to a SpringerOpen ${ }^{\circ}$ journal and benefit from:}

Convenient online submission

- Rigorous peer review

- Immediate publication on acceptance

- Open access: articles freely available online

- High visibility within the field

- Retaining the copyright to your article

Submit your next manuscript at $\boldsymbol{s p r i n g e r o p e n . c o m ~}$ 